\title{
Explotación minera y sus impactos ambientales y en salud. El caso de Potosí en Bogotá
}

\author{
Mining and its health and environmental impacts. The case of Potosí \\ in Bogotá \\ Ángela Marcela La Rotta Latorre', Mauricio Hernando Torres Tovar ${ }^{\mathbf{2}}$
}

RESUMO Bajo el modelo capitalista global este trabajo explora el vínculo entre la explotación minera y sus impactos dentro de los campos del medio ambiente y la salud, tomando el caso de la minería de materiales de construcción en Bogotá. El estudio es de carácter descriptivo cualitativo y sus fuentes provienen de revisión literaria, observación y percepciones por parte de los lugareños. Gracias a esta investigación se evidenciaron alteraciones en el estado de salud física y mental de la población, su tejido social y daños al ambiente; lo que cuestiona este modelo de desarrollo y sus patrones que ponen en riesgo la supervivencia, que va de la mano con el surgimiento de serios conflictos medioambientales, evidenciando la relación del ambiente tanto con la salud como con dinámicas socio-culturales.

PALAVRAS-CHAVE Minería. Salud. Ambiente. Desarrollo económico.

\begin{abstract}
Under the global capitalist model this paper explores the link between mining and its environmental and health impacts, taking the case of mining of building materials in Bogotá. The study is descriptive qualitative and its information comes from literature review, observation and perceptions of inhabitants. From this search alterations were found in the physical and mental health of the population, its social net, and environmental damage, questioning the current development model and its patterns that put at risk the survival and goes together with the emergence of serious environmental conflicts that show the relationship of the environment with socio-cultural dynamics and health.
\end{abstract}

KEYWORDS Mining. Health. Environment. Economic development.

1 Universidad Nacional de Colombia, Facultad de Medicina - Bogotá, Colômbia.

amlal@unal.edu.co

2 Universidad Nacional de Colombia, Facultad de Medicina, Departamento de Salud Pública - Bogotá, Colômbia.

mhtorrest@unal.edu.co 


\section{Introducción}

Actualmente, las dinámicas globales obedecen al modelo capitalista de producción a gran escala, acumulación, derroche $\mathrm{y}$ despojo. Éste modelo, a su vez, está soportado en el necesario consumo de productos, bienes y servicios que se ha ido incrementando con el auge de la tecnología, el crecimiento demográfico y las necesidades artificiales que crea el sistema productivo. Todos estos productos requieren de diversos recursos naturales para su producción, y para lograr satisfacer la demanda se ha recurrido a la explotación de múltiples territorios llevando a una destrucción sin precedentes.

Obedeciendo a este comportamiento mundial, el modelo extractivista se ha instaurado en Colombia como una de las principales actividades económicas, sin embargo, las consecuencias ambientales, sociales, culturales y económicas, debidas a la pobre regulación, el escaso control, la falta de presencia del Estado y la protección de intereses particulares producidas por estas actividades extractivas principalmente minero-energéticas han puesto en evidencia complejos conflictos de carácter socioambiental que repercuten profundamente en las dinámicas poblacionales y que comienzan a ser motivo de preocupación debido a los efectos que se vislumbran en las condiciones de vida, salud y el tejido social propio de las poblaciones cercanas a los lugares donde se desarrollan estos proyectos de explotación o de las que se han visto envueltas, directa o indirectamente, en los mismos.

En Colombia, La Ley 685 (Código de Minas) prácticamente coloca al Estado como un observador en cuanto a la actividad minera, mientras que incentiva la inversión privada, lo cual se ratifica en los Planes de Desarrollo Minero desde el año 2002 hasta la actualidad (INSUASTY RODRÍGUEZ; GRISALES; GUTIERREZ, 2013). En cuanto al sector ambiental la situación no es diferente; hasta hace poco menos de 10 años se entregaron cientos de títulos mineros para la explotación de áreas protegidas, lo que ocasionó enormes daños ecológicos y sociales, esto debido a que ni el Ministerio de Ambiente y Desarrollo Sostenible ni las corporaciones autónomas regionales, han definido en su totalidad el régimen de uso de las zonas que les corresponden, desde las reservas forestales hasta los humedales (SALAMANCA, 2013).

Este panorama crea un ambiente de 'confianza inversionista' que atrae el capital de diversas multinacionales para la explotación de los territorios, confrontando los intereses de las mismas con los del Estado y con los de las diversas comunidades que los habitan, comunidades que generan resistencia al defender sus recursos naturales y la salud de su población.

Estas problemáticas afectan principalmente a poblaciones vulnerables como los indígenas o campesinos, al vivir en tierras estratégicas (INSUASTY; GRISALES; GUTIERREZ LEÓN, 2013). Desafortunadamente, a las comunidades o no se les informa acerca de los proyectos mineros o se les informa mal o los intereses económicos juegan un papel clave. Esto es claro en el mecanismo de consulta previa según la Contraloría, ya que

[...] en el proceso de otorgamiento de los títulos mineros no se tiene en cuenta la consulta previa que es un derecho fundamental de las comunidades étnicas, pues se trata de un mecanismo básico para preservar su integridad étnica, social, económica y cultural, y para asegurar su supervivencia como grupos sociales, tal como la Corte Constitucional lo ha expresado en ocasiones reiteradas. (SALAMANCA, 2013, P. 25).

Existen múltiples ejemplos de este modus operandi para el otorgamiento de títulos mineros en Colombia. El Cerrejón en la Guajira, Cerro Matoso, el páramo de Santurbán, Caramanta, Marmato, Cajamarca, sólo por mencionar algunos.

Evidentemente, estas problemáticas $\mathrm{y}$ conflictos, unos ya existentes y posteriormente exacerbados por la minería, otros generados por la presencia de la misma, obedecen a una serie de factores que convergen 
para dar paso a un clima de tensión, incertidumbre y vulnerabilidad en las comunidades afectadas que desemboca luego en profundos daños sociales, ambientales, sanitarios y económicos. Como lo expresa la Contraloría General de la República

La presencia de complejos mineros en los territorios puede exacerbar situaciones como las antes descritas (...pauperización, confinamiento en el territorio, desplazamiento para satisfacción de necesidades básicas) o generar nuevos conflictos de pérdida de vigencia de derechos constitucionales e imposibilidades para que las poblaciones puedan disfrutar de una vida digna. (SALAMANCA, 2013, P. 58).

En el marco de esta dinámica nacional el presente trabajo explora los vínculos entre la explotación minera y sus impactos al ambiente y a la salud, tomando como ejemplo demostrativo el estudio de caso de la minería de materiales de construcción en Bogotá, específicamente en el barrio Potosí de la localidad de Ciudad Bolívar, donde la presencia de zonas de explotación minera ha dado paso a conflictos entre la comunidad, las entidades gubernamentales y los empresarios ubicados en este territorio, a causa de los efectos producidos tanto en el ambiente como en la salud de la población.

\section{Material y métodos}

Este estudio es de carácter cualitativo descriptivo. Primero se realizó una búsqueda bibliográfica en torno a la temática minera, específicamente de los procesos extractivos de materiales de construcción y sus efectos en el ambiente y la salud, de su legislación y del estado de la actividad minera en Colombia, en la capital y en Ciudad Bolívar, localidad de Bogotá, territorio concreto a estudiar.

Posteriormente se analizaron datos y estadísticas de informes provenientes de entidades gubernamentales, tanto nacionales como locales, referentes al estado de la actividad minera en Bogotá, a los impactos generados por la misma y al análisis general del estado de la población de la localidad de Ciudad Bolívar en conjunto con datos del último diagnóstico realizado por el Hospital Vista Hermosa, hospital de referencia de esta localidad. Igualmente se analizaron algunos indicadores de la localidad presentados por los observatorios de ambiente y salud ambiental de la Alcaldía Mayor de Bogotá, todo esto con el fin de caracterizar la población y su entorno.

Luego se recopilaron notas de prensa que permitieron complementar la información acerca de los actos de la comunidad y las dinámicas generadas entre los actores del conflicto socioambiental en el territorio de Potosí.

Por último, se realizó una visita al territorio guiada por integrantes de la Mesa Ambiental 'No le Saque la Piedra a la Montaña', habitantes del barrio, quienes han trabajado en la construcción de una estrategia para frenar la minería en el sector, para conocer, a partir de testimonios, la percepción de la comunidad frente a esta. Los nombres de los entrevistados fueron alterados para proteger su identidad.

\section{Resultados}

La ciudad de Bogotá, territorialmente, está divida en 20 localidades, y concretamente la localidad de Ciudad Bolívar se encuentra al sur de Bogotá, siendo la tercera más extensa de la ciudad con 13,000.2 hectáreas, de éstas 9,608.4 hectáreas pertenecen a zona rural y 3,239.8 a zona urbana con aproximadamente 713,764 habitantes (BOGOTÁ, 2017; BOGOTÁ, 2011A). En esta localidad se encuentra el Relleno Sanitario Doña Juana y el Parque Minero Industrial Mochuelo que pasa por cuatro veredas de la localidad.

El barrio Potosí se encuentra localizado en la parte más alta de uno de los cerros de la localidad, en su costado suroccidental. La estructura ecológica de la localidad posee 8 Áreas Forestales Distritales y el Parque Ecológico Distrital de Montaña (BOGOTÁ, 2013A). 
Figura 1. Ubicación de Ciudad Bolívar en Bogotá

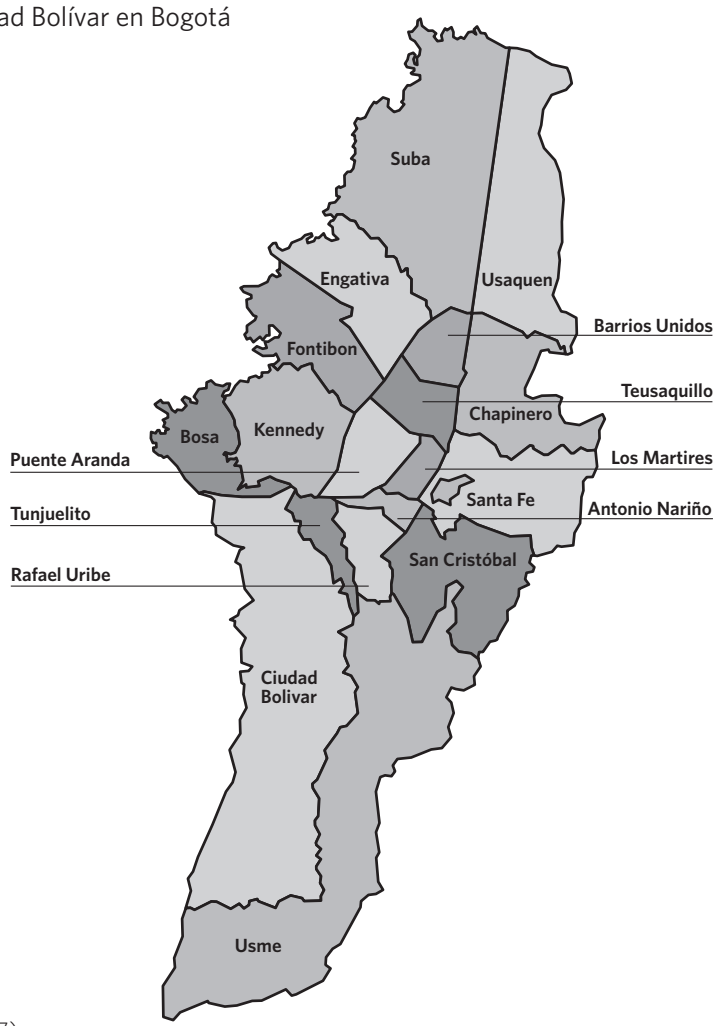

Fuente: MCS en Contacto (2017).

La población de esta localidad es, en general, de bajos recursos económicos según la estratificación de la ciudad, en tanto históricamente esta ha sido una zona a la que llega población migrante y desplazada en
Colombia y de sectores obrero-populares. La tabla 1 presenta datos que ayudan a su caracterización, comparándola con la población general de Bogotá, especialmente en cuanto a indicadores de pobreza y condiciones de vida.

\begin{tabular}{|c|c|c|c|c|c|c|}
\hline \multirow{4}{*}{$\begin{array}{l}\text { Parametro } \\
\text { Inseguridad Alimentaria }\end{array}$} & \multicolumn{2}{|l|}{ Descripción } & \multicolumn{2}{|c|}{ Ciudad Bolívar } & \multicolumn{2}{|c|}{ Bogotá } \\
\hline & Porcentaje de Hogares con Inseguridad & Leve & $35.8 \%$ & $26.8 \%$ & $24.3 \%$ & $18.2 \%$ \\
\hline & Alımentarıa (lotal) & Moderada & & $8.5 \%$ & & $5.2 \%$ \\
\hline & & Severa & & $0.5 \%$ & & $0.9 \%$ \\
\hline Percepción de pobreza & \multicolumn{2}{|c|}{ Porcentaje de la población que se considera pobre } & & $33.9 \%$ & & $24.3 \%$ \\
\hline \multirow[t]{2}{*}{ Pobreza por ingresos } & \multicolumn{2}{|l|}{ Personas pobres por ingresos } & & $32.2 \%$ & & $17.3 \%$ \\
\hline & \multicolumn{2}{|l|}{ Personas en indigencia por ingresos } & & $5.9 \%$ & & $4.0 \%$ \\
\hline \multirow[t]{2}{*}{ Necesidades Básicas Insatisfechas (NBI) } & \multicolumn{2}{|l|}{ Pobreza por NBI } & & $9.8 \%$ & & \\
\hline & \multicolumn{2}{|l|}{ Miseria por NBI } & & $0.7 \%$ & & \\
\hline Coeficiente de GINI & \multirow{2}{*}{\multicolumn{2}{|c|}{ Miseria por NBI }} & & 0.380 & & 0.542 \\
\hline Índice de Pobreza Multidimensional & & & & $6.2 \%$ & & $3.2 \%$ \\
\hline
\end{tabular}

Fuente: Bogotá (2011b) 
Se observa que en comparación con Bogotá esta localidad se encuentra, en su mayoría, por encima de los valores promedio para la ciudad, lo que permite situarla dentro de las localidades con más segregación, desigualdad y marginación.

Por otra parte, el Observatorio de Salud Ambiental y el Observatorio Ambiental de Bogotá proporcionan datos de acuerdo al registro periódico de indicadores de los componentes estudiados en salud ambiental (aire, ruido, calidad de agua y saneamiento básico, entre otros) y de los componentes ambientales propiamente dichos, recogidos en las 20 localidades de la ciudad (BOGOTÁ, 2017).

Según el último diagnóstico local del Hospital Vista Hermosa, las enfermedades respiratorias se encuentran dentro de las primeras causas de consulta externa y urgencias en todas las edades (BOGOTÁ, 2013B). Teniendo en cuenta lo anterior, en la siguiente tabla se presentan los datos de algunos indicadores correspondientes a la calidad del aire en la localidad de Ciudad Bolívar con respecto a la de la ciudad.

Tabla 2. Indicadores de salud ambiental relacionados con la calidad del aire en Ciudad Bolívar

\begin{tabular}{|c|c|c|c|c|c|c|c|}
\hline \multirow{2}{*}{ Parametro } & \multirow{2}{*}{ Indicador } & \multicolumn{2}{|c|}{2013} & \multicolumn{2}{|c|}{2014} & \multicolumn{2}{|c|}{2015} \\
\hline & & $\mathrm{CB}$ & BOG & $C B$ & BOG & $\mathrm{CB}$ & BOG \\
\hline \multirow[t]{4}{*}{ Calidad del Aire } & $\begin{array}{l}\text { Casos salas ERA en menores de } 5 \text { años (meses: } \\
\text { ene-mar) }\end{array}$ & $\begin{array}{r}1017 \\
10.3 \%\end{array}$ & 9872 & $\begin{array}{r}1238 \\
10.4 \%\end{array}$ & 11862 & $\begin{array}{r}722 \\
7.1 \%\end{array}$ & 10099 \\
\hline & $\begin{array}{l}\text { Prevalencia de Sibilancias en menores de } 5 \text { años } \\
\text { posiblemente asociado a Material Particulado PM10 }\end{array}$ & \multicolumn{2}{|c|}{$\begin{array}{r}\text { Sin datos para la } \\
\text { localidad }\end{array}$} & 16.6 & 13.1 & 19.0 & 13.2 \\
\hline & $\begin{array}{l}\text { Prevalencia de ausentismo escolar por enfermedad } \\
\text { respiratoria en menores de } 14 \text { años }\end{array}$ & 11.9 & 14.7 & 13.7 & 17.9 & 22.0 & 19.3 \\
\hline & $\begin{array}{l}\text { Quejas atendidas por exposición a contaminación del } \\
\text { aire en la localidad }\end{array}$ & & 12.0 & & 11.0 & & 1.0 \\
\hline
\end{tabular}

Fuente: Bogotá (2017).

CB: Ciudad Bolívar; BOG: Bogotá; ERA: Enfermedad Respiratoria Aguda; PM10: Material Particulado con diámetro menor a 10 $\mu$ m.

En la tabla 2 se observa la alta prevalencia de sibilancias en menores de 5 años de la localidad comparada con los demás menores de la ciudad. El porcentaje de los casos totales para los meses de enero a marzo corresponde en 2013 y 2014 a aproximadamente el 10\% del total de la ciudad. Teniendo en cuenta que son 20 localidades se considera un alto número de casos aportados por una sola localidad, cifra que disminuye en el último año sin una causa descrita en los indicadores. La prevalencia de ausentismo escolar aumenta en el último año por encima de los valores de la ciudad y las quejas por exposición a contaminación del aire en 2013 y 2014 sólo fueron superadas por otras dos localidades (Bosa y
Kennedy), recibiendo de 2 a 5 quejas en las demás localidades.

Esto último concuerda con el promedio de PM10 (material particulado de diámetro menor a $10 \mu \mathrm{m}$ ) según el Plan Ambiental Local (PAL) de Ciudad Bolívar para 2013-2016, donde las mayores concentraciones en la ciudad, más allá del límite permitido, se encuentran dentro de las localidades Ciudad Bolívar, Bosa y Kennedy (BOGOTÁ, 2012), como lo muestra la figura 2. Esto se debe, fundamentalmente, a la confluencia de varias avenidas de alto tráfico en estas localidades y la presencia de industrias contaminantes, entre ellas las de carácter extractivo. 
Figura 2. Concentración PM10, promedio anual 2011 en Bogotá

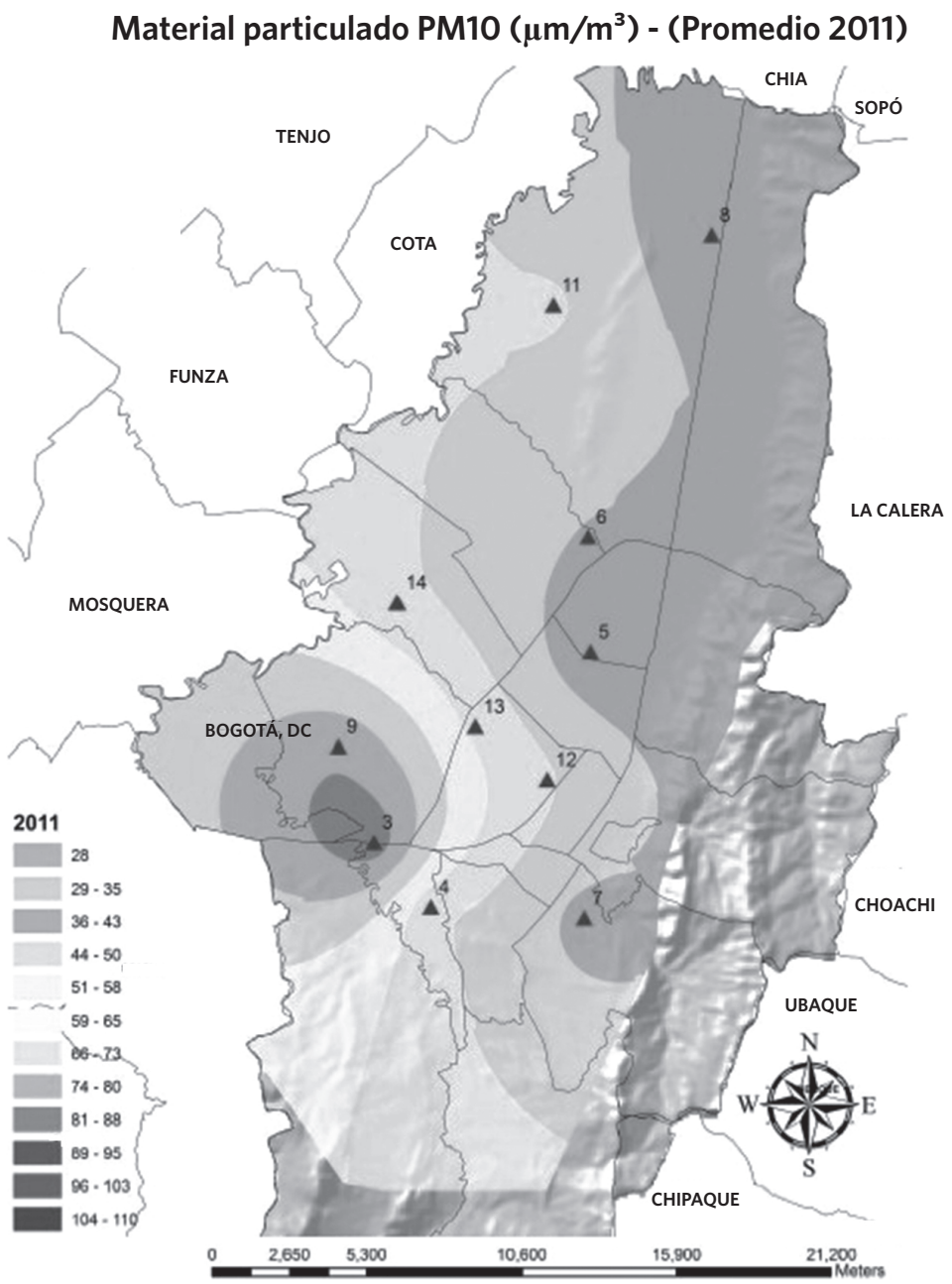

Fuente: MCS en Contacto (2017)

Igualmente, se reportan valores por encima de los límites para las concentraciones de PM2.5 (Material Particulado con diámetro menor a $2.5 \mu \mathrm{m}$. Límite anual $25 \mu \mathrm{g} / \mathrm{m} 3$, promedio anual encontrado con exceso de $365 \mu \mathrm{g} /$ m3) y para los niveles de partículas suspendidas totales (PST) (límite anual $100 \mu \mathrm{g} / \mathrm{m} 3$, valor encontrado $144 \mu \mathrm{g} / \mathrm{m} 3$ ) (BOGOTÁ, 2013A). A la luz de estos hallazgos es necesario observar la situación minera de la ciudad y la localidad según los impactos que ésta genera en el ambiente y la salud, teniendo en cuenta que en esta zona se realiza extracción de materiales de construcción y uno de sus principales efectos directos es la contaminación del aire por emisión de material particulado.

La tabla 3 muestra la situación de los predios en la zona urbana de Bogotá. Los predios mineros registrados en la ciudad hasta el año 2013 por la Contraloría de Bogotá se dividen en 6 localidades, encontrándose en mayor número en Ciudad Bolívar y Usme. Adicionalmente, se observa el estado legal de estos predios, la existencia o no de Planes de Manejo Ambiental (PMA) y el estado de actividad de las minas. 
Tabla 3. Estado de predios mineros en zona urbana de Bogotá

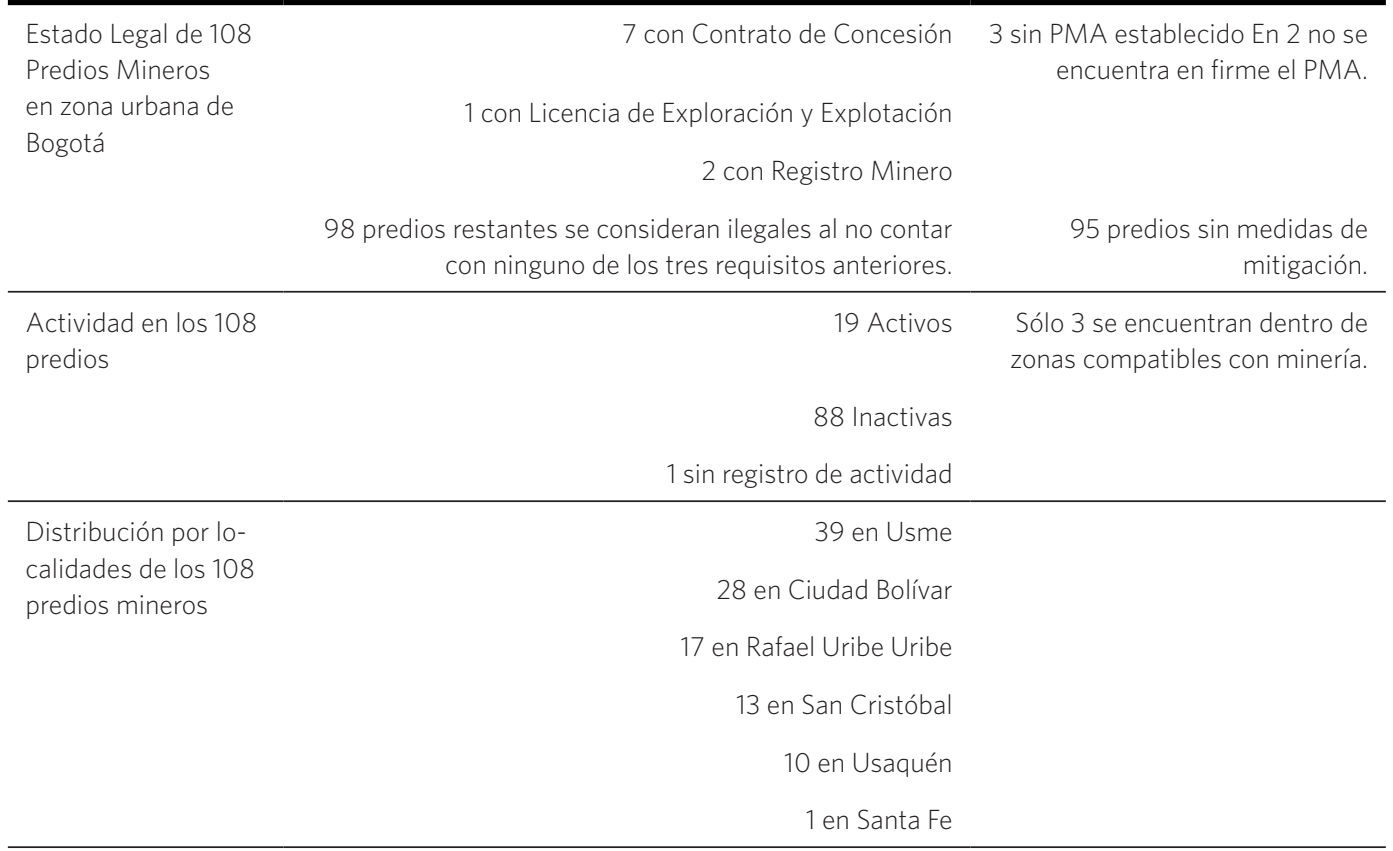

La SDA no especifica para ninguno de los 108 predios si se encuentran o no dentro de áreas compatibles con extracción minera.

Fuente: Salamanca (2013).

PMA: Planes de Manejo Ambiental; PMRRA: Planes de Recuperación y Restauración Morfológica y Ambiental; SDA: Secretaria Distrital de Ambiente.

Es evidente la escasa regulación hacia esta industria extractiva si se tiene en cuenta que el $90 \%$ de los predios no cuentan con los requisitos legales para operación, de éstos el $96 \%$ no tiene medidas de mitigación y de los legales o no tienen PMA o se encuentra en construcción. Esta ilegalidad se explica, según la Comisión Económica para América Latina y el Caribe (Cepal), por predios que han pertenecido a familias dedicadas a la explotación de estos materiales y por erróneas u obsoletas interpretaciones de la ley que amparan hoy a algunos predios (CEPAL, 2004). Igualmente es notorio que la cantidad de los predios mineros en la ciudad converge, en su mayoría, dentro de las localidades de más bajos recursos.

En el caso específico de Potosí existe un predio de 169 hectáreas con cuatro frentes de explotación minera (HERRERA DURÁN, 2015) dedicados a la extracción de arenas amarillas, roca y recebo. A 200 metros hacia el noroccidente se encuentra el colegio distrital Instituto Cerros del Sur y aproximadamente a la misma distancia inicia la línea de hogares que componen el barrio. Este predio se encuentra dentro de los enumerados como legales por el informe de la Contraloría, sin embargo, no se encuentra dentro de zona habilitada para minería por la Resolución 222 de 1994. Este predio no tenía PMA establecido ni acción frente al requerimiento del mismo y para el año 2013 no había registro del último concepto técnico, ya sea porque no se registró o porque no se realizó hasta ese momento. Igualmente, en este y otros dos predios, la vigencia de los contratos de concesión excede los 30 años de ley (de diciembre 26 de 1991 a mayo 9 de 2026) (SALAMANCA, 2013).

Para este predio, la Secretaria Distrital de Ambiente (SDA) emitió el Auto No 01052 con fecha del 4 de mayo de 2015. En este 
documento se registran las acciones hacia el predio minero iniciadas desde el año 2000 por un oficio enviado a la Corporación Autónoma Regional (CAR) por la Junta de Acción Comunal de Arborizadora Alta Sector Sena, barrio aledaño a Potosí, "debido a la grave contaminación a la población del sector, entre otras afectaciones" (BOGOTÁ, 2015, P. 2).

Aquí se documentan los resultados de las visitas técnicas realizadas por la SDA los días 9, 13 y 18 de marzo de 2015, encontrando varios hallazgos: zonas de explotación fuera de las áreas del contrato; zonas de erosión dejando bloques de arenisca con alto riesgo de caída que se encuentran en las áreas no permitidas; acopios de hasta 2 metros de altura de suelo orgánico y recebo, localizados en la parte alta de los taludes favoreciendo procesos de remoción en masa; afectación de acuíferos; remoción y disposición inadecuada de suelos negros en laderas y zona de ronda de la quebrada Tibanica; inexistencia de manejo de aguas de escorrentía y sedimentos, que además de contaminación de aguas y suelos puede generar procesos de remoción en masa; emisión de material particulado por el viento debido a falta de cobertura vegetal, afectando zonas aledañas (BOGOTÁ, 2015).

Igualmente, se encontró afectación del ecosistema subxerofítico de la zona, ecosistema que

se ha identificado como estratégico para el Distrito, debido a sus características únicas de flora y fauna, los extensos pastizales xerófilos constituyen un alto potencial para la captura de $\mathrm{CO} 2$ y por la gran diversidad endémica que sustenta (BOGOTÁ, 2015, P. 15),

sin hallar planes de reforestación, generando además un impacto visual negativo.

Dado lo anterior se consideró que las actividades se estaban realizado fuera de zona compatible con minería y además sin instrumentos de manejo y control ambiental por lo que se procedió al cierre preventivo de la explotación y solicitud e implementación del Plan de Mitigación Ambiental/Plan de Recuperación y Mitigación Ambiental (PMA/PMRA) (BOGOTÁ, 2015). Debido a este concepto y a la presión ejercida por la comunidad y los integrantes de la Mesa Ambiental, a la fecha, la cantera lleva más de un año inactiva y en espera de que se logre su cierre definitivo.

Durante el tiempo en que la mina estuvo abierta, y aún hoy en día, la población manifestó varias quejas en cuanto a su salud y su entorno. La mayoría debidas al paso de volquetas que subían a la mina y la gran cantidad de ruido y polvo que estas generaban a su paso, polvo originado tanto por el paso de los vehiculos por calles sin pavimentar como por el material de carga (arena), provocando problemas respiratorios, especialmente en los más pequeños: "Los pulmones. Mucha gripa. [...] Los niños mantenían muy enfermos. El tierrero era demasiado" (Rosa, 34 años. Habitante del barrio Potosí); y de contaminación auditiva por la llegada de las volquetas a tempranas horas de la mañana:

El ruido, desde las 3 de la mañana pasando las volquetas, dañando nuestras calles. (Teresa, 68 años. Habitante del barrio Potosí).

Todos los días a las 3:30, 4 de la mañana, afectaba mucho, llegaban pitando. Hasta cerraron la entrada que hay allí y llegaban a pitar. (Pedro, 42 años. Habitante del barrio Potosí).

También, el daño evidente a la montaña y el componente ambiental que afectó, incluso, sus dinámicas de grupo:

La montaña, pues, tan linda que era. Nosotros íbamos a pasear por allá y ċahora qué? No, ya no podemos ir por allá. (Teresa, 68 años. Habitante del barrio Potosí).

En un tiempo incluso también existía la quebrada, y allá íbamos a lavar. Era una caminata hasta 
allá. Y todos con su bultico de ropa al hombro, iban allá, se hacía almuerzo, y por la tarde volver. Arriba también había un nacimiento de agua que era el que repartía el agua para acá. Se cogía el agua en tinas. Y también se secó. (Pedro, 42 años. Habitante del barrio Potosí).

En cuanto a las oportunidades de empleo no se consiguió realizar ninguna entrevista a las personas que trabajaban en la mina ya que abandonaron el territorio luego del cierre de la misma. Sin embargo, los habitantes refieren que fueron muy pocos los que encontraron esta actividad como un medio de empleo, solo en contados casos eran llamados a un empleo ocasional o realizaban algunas ventas ambulantes en los alrededores.

Finalmente, la población habitante de este barrio ve a la minería como la causa de las distintas problemáticas:

[...] el problema no eran las volquetas, no era el polvo, el problema estructural es la minería, que es la que genera todo el deambular de volquetas y todo eso. (Integrante de la Mesa Ambiental 'No le saque la piedra a la montaña')

y están generando soluciones como la concientización de la comunidad con la Escuela Ambiental del sector y el trabajo desde el colegio Instituto Cerros del Sur, e incluso la propuesta de creación del Parque Metropolitano Cerro Seco, que proteja las montañas de la explotación y la expansión urbana hacia el sur de la ciudad.

\section{Discusión}

Desde 1995, año en que fue otorgado el título minero en el sector de Potosí, los habitantes de la zona han vivido en las cercanías de la actividad extractiva al ser el barrio que se encuentra más próximo a ésta. Este tipo de minas dentro de la ciudad tienen una característica común: están ubicadas en zonas aledañas a barrios de estratos bajos, barrios que bien ya se encontraban allí al momento de iniciar la actividad, o que se han ido construyendo alrededor de la misma. Igualmente, en barrios que se han formado, de manera ilegal, dentro de las canteras abandonadas. En el caso de Ciudad Bolívar, en los años cuarenta, los lotes eran comprados a muy bajo precio por personas que llegaban a la ciudad desde el campo desplazadas por la violencia y con los años se fue concentrando allí la población marginada. Con el progresivo crecimiento desordenado de la ciudad hacia el sur fueron surgiendo nuevos barrios que seguían manteniendo esta particularidad económica (BOGOTÁ, 2011B). Consecuentemente con este crecimiento poblacional la industria minera de los materiales de construcción fue en aumento, instaurándose en suelos clave por su composición y su cercanía a la ciudad (GUZMÁN CASTIBLANCO, 2015), con lo que se distribuirían los minerales de manera local.

Como se observó en la tabla 1, la población de esta localidad reúne las características de las zonas de marginación y pobreza de la ciudad. Estas características se han visto en diversas zonas de Sur América y ha conferido a estos sectores toda clase de vulnerabilidades como lo expresan Breilh y sus colegas en el siguiente fragmento:

El proceso histórico que vivió el continente americano ha promovido un conjunto de vulnerabilidades e inequidades en la región e impuesto un enorme sufrimiento, principalmente a las sociedades de Centroamérica, el Caribe y América del Sur, donde se observa un crecimiento desordenado de las ciudades y una facilidad de instalación de industrias contaminantes, lo que promueve toda suerte de exclusiones, que se manifiestan en el aumento de la violencia, las amenazas a los ecosistemas y a la biodiversidad, la contaminación del suelo, el aire y las aguas, y la vulnerabilidad regional a los cambios climáticos. (BREILH ET AL., 2010, P. 23).

Este escenario desemboca en una serie de situaciones que ponen en riesgo el entorno, 
la salud y el mismo modo de vida de la población, como lo han denunciado reiteradamente los habitantes de Potosí en medios de comunicación (HERRERA DURÁN, 2015; APONTE, 2015).

De acuerdo a los indicadores presentados en los resultados del Hospital Vista Hermosa y el Observatorio de Salud Ambiental, las enfermedades respiratorias son comunes en este sector en individuos de todas las edades y constituyen un gran número de consultas a urgencias y consulta externa. La percepción de la comunidad concuerda con esta visión, haciendo énfasis en la afectación a los habitantes más jóvenes.

Ahora que la cantera lleva un año cerrada manifiestan mejoría en su percepción de salud y su vida comunitaria, pero siguen haciendo llamados a una fuerte regulación en cuanto a esta práctica. Aquí el papel de las entidades competentes es fundamental, pero insuficiente para atender estas problemáticas. Frente a esta visión la Contraloría de Bogotá formula una posición importante. Cuando se expidió el informe de auditoría a la SDA en 2013, se había ordenado el cierre de 11 industrias mineras, 7 años atrás, sin embargo, sin exitoso cumplimiento

Situación que se deriva de la falta de gestión y control por parte de la Secretaria Distrital de Ambiente, a las actividades mineras desarrolladas en el Distrito Capital, lo que trae como consecuencia que se sigan realizando actividades de explotación mineras en zonas no compatibles para desarrollar la minería y que además no se implementen las medidas de prevención, mitigación, corrección para la recuperación del terreno, lo que puede conllevar a que se afecte gravemente el ambiente y la salud de la población. (SALAMANCA, 2013, P. 6).

El Programa de las Naciones Unidas para el Medio Ambiente (Pnuma) y la Cepal concuerdan en este punto, donde la debilidad normativa hacia esta industria es una de las razones que impide que se haga un buen uso de la misma, teniendo en cuenta su crecimiento debido a la urbanización de la región, y reconocen los múltiples efectos ambientales y sociales al ser una actividad que se instaura en la periferia de las ciudades, donde se da uso final a los productos ya que es una industria predominantemente local. Esto hace que la actividad se torne más 'cercana' y deba ser de mayor control, exigencia y resistencia por parte de los pobladores (CEPAL, 2004; PNUMA, 2013).

Por esta 'cercanía' de la actividad y por la naturaleza de la misma, los efectos que produce son igualmente notorios. Se elimina la capa vegetal y con ella el hábitat de múltiples especies, como lo describe en párrafos anteriores el concepto técnico de la SDA, o se arboriza el territorio con especies que no son nativas, alterando el equilibrio del ecosistema; aumenta el material particulado contaminando aire y agua; se produce contaminación del aire por gases de las chimeneas y material particulado por la combustión en los hornos; contaminación auditiva por ruido de maquinaria, vehículos y explosiones, y destrucción de las vías por los mismos (CEPAL, 2004). Además, un impacto visual negativo muy importante (BOGOTÁ, 2010). Sumado a esto, desde los años 70, en América Latina los ingresos por explotación de recursos primarios han ido en disminución. Y en el caso particular de Bogotá se ha denunciado que la ciudad no ha recibido regalías por esta actividad, ni siquiera por parte de las grandes empresas, o si se venían pagando, en los últimos años dejaron de percibirse (HERRERA DURÁN, 2016; GUZMÁN CASTIBLANCO, 2015).

El suelo es un gran motivo de preocupación con el paso del tiempo y su explotación. Pierde casi en su totalidad la materia orgánica, se vuelve inestable, cambia sus propiedades físico-químicas, pierde su productividad e indirectamente contamina el aire y las fuentes de agua circundantes por la acumulación de materiales tóxicos en su substrato y por el proceso de erosión y desertificación que le sigue (BECERRIL ET AL., 2007).

En Colombia, varias regiones están en 
proceso de desertificación debido a la disminución de la precipitación por el fenómeno del cambio climático que vemos en la actualidad, entre ellas las más afectadas serán la Andina y el litoral Caribe, zonas con la mayor demanda de suelos por la alta densidad poblacional. Por lo tanto, preocupa que se desarrollen proyectos mineros en estas regiones teniendo en cuenta lo anterior y considerando el impacto en el recurso hídrico ya que, aparte de la contaminación descrita, se utilizan grandes cantidades de este líquido en el proceso de obtención del material (SALAMANCA, 2013) y para mitigar la emisión del material particulado durante la extracción.

Todas estas preocupaciones anteriores son notables y la afectación del paisaje da cuenta de ello. La actividad en estos predios se realiza sin prever un uso futuro para el mismo y perjudica grandes extensiones de suelo dejándolas erosionadas y estériles. Estas zonas luego quedan excluidas de los planes de ordenamiento territorial y son ocupadas posteriormente por asentamientos informales los cuales quedan ubicados en zonas de alto riesgo al no realizarse una recuperación morfológica del espacio (GUZMÁN CASTIBLANCO, 2015). Esto hace parte de una percepción se extiende entre la comunidad del barrio Potosí, quienes han manifestado un cambio drástico en el paisaje desde que comenzó la explotación, dejándolos sin uno de sus principales sitios de actividad cultural y recreativa, ya que ahora el terreno está deteriorado y en manos de terceros, sumándose a esto la desaparición del rio que pasaba por el sector (APONTE, 2015).

En este punto es importante mencionar los pasivos ambientales mineros (PAM), los cuales, a diferencia de otros países de la región, no están reglamentados como tal en Colombia, pero hacen referencia a

un área donde existe la necesidad de restauración, mitigación o compensación por un daño ambiental o impacto no gestionado, producido por actividades mineras inactivas o abandonadas que pone en riesgo la salud, calidad de vida o bienes públicos o privados (ARANGO ARAMBURO, 2012),

definición que se usa en la mayor parte de Suramérica.

En teoría, las minas deben tener un proceso al momento de su cierre o en período de inactividad, pero esto en muchos casos no sucede y no se mitigan o reparan los daños ambientales dejando zonas contaminadas y riesgos de salud para la población (ARANGO ARAMBURO, 2012). Esto sucede no solo por debilidad normativa, sino por falta de compromiso ético, social y ambiental de los mismos explotadores de las minas (CEPAL, 2014; BOGOTÁ, 2011B).

Éstos PAM reúnen todos los componentes contaminantes, ambientales, paisajísticos, sociales y económicos que quedan después de la actividad minera. Sin embargo, se aclara que sólo aquellos impactos que generan un riesgo para la salud o el ambiente son considerados PAM, ya que posteriormente generarán una obligación económica debida a ese riesgo; son los mismos 'sitios huérfanos' o 'deudas ambientales' en otros países del mundo. En Colombia hay mecanismos para la reparación de estos pasivos, sin embargo, no hay un control y un seguimiento estricto por parte de los entes competentes (ARANGO ARAMBURO, 2012). Estos pasivos los terminan asumiendo, al menos en principio, las comunidades más vulnerables que están más próximas al escenario, constituyendo un claro ejemplo de injusticia ambiental (ARRIAGA LEGARDA; PARDO BUENDİA, 2011).

Teniendo en cuenta todo lo enunciado hasta ahora, el proceso salud-enfermedad en esta población tendrá unas características y un desarrollo propios, ya que esta dinámica se produce a diferentes niveles (ambiental, social, económico, político) y sus problemas han sido explicados en un entorno particular (CASTELLANOS, 1990) dado por el medio ambiente en el que habitan, sus condiciones económicas bajas y la presencia de industria 
extractiva escasamente regulada. Por lo tanto, la asociación que hace la comunidad entre las enfermedades respiratorias, el material emitido durante el transporte de los productos de la mina, la perturbación auditiva por los vehículos, la falta del lugar para actividad recreativa y el visible deterioro paisajístico que genera cierta perturbación emocional, da cuenta de este proceso salud-enfermedad y de otros similares que se pueden estar viviendo en otros barrios de la ciudad.

Si las industrias de minería de materiales de construcción están ubicadas en otras zonas marginadas de la ciudad, como se vio en la distribución por localidades, cabe pensar que las demás comunidades ubicadas en dichas zonas están viviendo una problemática similar, obedeciendo a un fenómeno de reproducción social y a unos procesos de determinación social que

no actúan como agentes biológicos-físicosquímicos en la generación de la enfermedad, no tienen especificidad etiológica, ni obedecen a una mecánica de dosis-respuesta. (ARELLANO; ESCUDERO; MORENO, 2008, P. 324)

pero que deben ser tenidos muy en cuenta al momento de pasar a la atención de la enfermedad y al crear estrategias de prevención, ya que

Estos factores configuran distintos modos de pensar y de actuar, con una diversidad de significaciones que se atribuyen al hecho de estar sano o enfermo, donde aspectos como la edad, el sexo y las condiciones materiales de vida, tienen una multiplicidad de expresiones.

(SACCHI; HAUSBERGER; PEREYRA, 2007, P. 272)

Entonces, si la industria extractiva está causando o exacerbando estos escenarios, ¿cuál debe ser la solución? Es necesario plantearse la alternativa de una minería sustentable que no afecte o minimice sus efectos en el ambiente y, por consiguiente, en la salud, pero ¿̇es realmente posible? el acelerado ritmo de la economía actual hace pensar que no.

El mercado no garantiza que la economía encaje en la ecología, ya que el mercado infravalora las necesidades futuras y no cuenta los perjuicios externos a las transacciones mercantiles. (ALIER, 2008, P. 13).

Hacia el otro extremo está entonces la desaparición de la actividad extractiva en la ciudad. Como se mencionó antes, la minería de materiales de construcción es una industria local, sus productos se distribuyen en las cercanías del sitio de extracción. Si Bogotá aún sigue creciendo, aparentemente sin planes para detener la expansión, con qué materiales se realizarán las construcciones $\mathrm{y}$ vías planeadas. Es necesario entonces replantear el modelo de ciudad y frenar la expansión de la capital en pro de mejorar las condiciones ambientales y de salud de los que habitan en ella, en tanto megaciudades como Bogotá son ya consideradas inviables tanto desde el punto de vista social como ambiental. Y si esta expansión es inevitable es absolutamente necesario encontrar alternativas de materiales que no pongan en riesgo el medio ambiente ni la salud.

\section{Conclusiones}

El modelo de desarrollo actual global impone una serie de patrones que ponen en riesgo la supervivencia misma de la especie humana. La destrucción de incontables regiones del planeta a manos de los procesos de producción intensivos va en conjunto con la emergencia de serios conflictos socioambientales que ponen en evidencia la innegable relación del ambiente con las dinámicas socio-culturales y, en particular, con la salud.

Es necesario cuestionar, entonces, el modelo de ciudad de nuestra región y el modelo de desarrollo global, así como el mismo concepto de desarrollo. ¿Qué es para 
nosotros el desarrollo, qué queremos con el desarrollo? ¿Estamos dispuestos a cambiar nuestro modo de vida y nuestros patrones de consumo para encaminarnos en una dirección diferente de un desarrollo ligado más al vivir saludablemente, visión que le apuesta a una relación de respeto y equilibrio entre la sociedad y la naturaleza?

Sin duda, los estrechos vínculos de determinación de la salud hacia las condiciones ambientales, tal como se evidencian en el caso del barrio Potosí, en la ciudad de Bogotá, demandan a las ciencias de la salud fortalecer el campo de conocimiento de la salud ambiental, para entender plenamente porqué se dan los cambios ambientales, cuáles son sus razones estructurales y cómo estos cambios afectan la salud de grupos poblacionales, para plantear estrategias de intervención de índole normativa, de política pública, de carácter ecológico y epidemiológico y de control ciudadano a partir de una amplia participación social.

Al ser el proceso salud-enfermedad una construcción que se desarrolla dentro de un contexto sociocultural determinado, fuertemente relacionado con el ambiente y el territorio donde se da, resulta necesario tratarlo y entenderlo como un proceso complejo en el que se deben buscar las causas de un padecimiento más allá del componente biológico. Este concepto es, o debería ser, indispensable en el qué hacer del cuidado, protección y atención a la salud. Esto implica un fuerte compromiso social que, en últimas, es lo que caracteriza a la medicina y otras profesiones de la salud, pero además un cambio de paradigma y la apertura hacia el trabajo con otras disciplinas para lograr una intervención integral y efectiva, entendiendo que desde las profesiones de la salud se tiene la responsabilidad de ser garantes no sólo del bienestar, sino de calidad de vida para las poblaciones.

\section{Colaboradores}

Ángela Marcela La Rotta Latorre: contribución substancial para la concepción, la planificación, para el análisis y la interpretación de los datos; participación de la aprobación de la versión final del manuscrito. Mauricio Hernando Torres Tovar: contribución significativa en la elaboración del rascuño y la revi-sión crítica del contenido; participación en la aprobación de la ver-sión final del manuscrito.

\section{Agradecimientos}

A los integrantes de la Mesa Ambiental 'No le saque la piedra a la montaña' y a la comunidad del barrio Potosí. 


\section{Referencias}

ALIER, J. M. Conflictos ecológicos y justicia ambiental. Papeles de relaciones ecosociales y cambio global, Madrid, n. 103, p. 11-28, 2008.

APONTE, A. La mina que le 'sacó la piedra' a una localidad en Bogotá. Kienyke, [online]. Disponible en: $<$ http://www.kienyke.com/historias/la-mina-que-le-saco-la-piedra-una-localidad-en-bogota/>. Acceso en: 14 marzo 2017.

ARANGO ARAMBURO, M. Problemática de los pasivos ambientales mineros en Colombia. Revista Gestión y Ambiente, Bogotá, v. 15, n. 3, p. 125-133, 2012.

ARELLANO, O. L.; ESCUDERO, J. C.; MORENO, L. D.

C. Los determinantes sociales de la salud: una perspectiva desde el taller latinoamericano de determinantes sociales sobre la salud, Alames. Medicina Social, Bronx, v. 3, n. 4, p. 323-335, 2008.

\section{ARRIAGA LEGARDA, A.; PARDO BUENDIA, M.} Justicia ambiental: el estado de la cuestión. Revista internacional de sociología, Madri, v. 69, n. 3, p. 627-648, 2011.

BECERRIL, J. M. et al. Especies nativas de suelos contaminados por metales: aspectos ecofisiológicos y su uso en fitorremediación. Ecosistemas, Madri, v. 16, n. 2, p. 50-55, 2007.

BOGOTÁ. Contraloría de Bogotá, DC. Secretaria Distrital de Ambiente. Informe de auditoría gubernamental con enfoque integral - modalidad especial: gestión en el control y seguimiento de las explotaciones mineras de Bogotá del periodo de junio de 2008 a diciembre 31 de 2012. Bogotá: Secretaria Distrital, 2013a. Disponible en: <http://www.contraloriabogota.gov.co/intranet/contenido/informes/ AuditoriaGubernamental/H\%C3\%Albitat\%20y\%20 Ambiente/PAD_2013/Especial/AGEIME_SDA MINERIA.pdf >. Acceso en: 14 marzo 2017.

Observatorio de Salud Ambiental. Línea de aire, ruido y radiación electromagnética: indicadores calidad del aire por localidad y para el distrito capital.
Disponible en: <http://biblioteca.saludcapital.gov.co/ ambiental/index.shtml? $\mathrm{s}=\mathrm{j} \& \mathrm{v}=4 \& \mathrm{p}=21>$. Acceso en: 14 marzo 2017.

Secretaría de Cultura, Recreación y Deportes. Plan local de cultura. Localidad de ciudad bolívar. Bogotá: Secretaría de Cultura, Recreación y Deportes, 2011a. Disponible en: <http://www.bogota.gov.co/localidades/ciudad-bolivar>. Acceso en: 14 marzo 2017.

Secretaría Distrital de Ambiente. Auto $n^{\circ}$ 01052: por el cual se inicia un procedimiento sancionatorio ambiental y se toman otras determinaciones. Bogotá: Secretaría Distrital de Ambiente, 2015. Disponible en: $<$ http://190.27.245.106/BLA/resoluciones/AUTOS\%20 2015/1052.pdf >. Acceso en: 14 marzo 2017.

Secretaría Distrital de Ambiente. Plan ambiental local: localidad 19, ciudad bolívar - 20132016. Bogotá, 2012. Disponible en: <http://www. ambientebogota.gov.co/documents/10157/2883179/ PAL+CBol\%C3\%ADvar+2013-2016.pdf>. Acceso en: 14 marzo 2017.

Secretaría Distrital de Planeación. 21 monografías de las localidades: diagnóstico de los aspectos físicos, demográficos y socioeconómicos. localidad \#19 ciudad bolívar. Bogotá, 2011b. Disponible en: <http:// www.sdp.gov.co/portal/page/portal/PortalSDP/ InformacionEnLinea/InformacionDescargableUPZs/ Localidad\%2019\%20Ciudad\%20Bol\%EDvar/ Monografia/19\%20Ciudad\%20Bolivar\%20monografia\%202011.pdf>. Acceso en: 14 marzo 2017.

Secretaría de Salud. Diagnóstico local de salud ciudad bolívar 2012. Bogotá: Secretaria de Salud, 2013b. Disponible en: <http://www.hospitalvistahermosa.gov. co/web/node/sites/default/files/boletines_2013/diagnostico_local/Diagnostico_local_salud_CB_2012.pdf>. Acceso en: 14 marzo 2017.

BREILH, A. et al. La transición hacia un desarrollo sostenible y la soberanía humana: realidades y perspectivas en la región de las américas. In: GALVAO, L. A. C.; FINKELMAN, J.; HENAO, S. (Ed.). Determinantes 
ambientales y sociales de la salud. Washington, DC:

Organización Panamericana de la Salud, 2010. p. 17-31.

\section{COMISIÓN ECONÓMICA PARA AMÉRICA LATINA}

Y EL CARIBE (CEPAL). División de Recursos

Naturales e Infraestructura. Industria minera de los

materiales de construcción: su sustentabilidad en améri-

ca del sur. Santiago: Cepal, 2004.

CASTELLANOS, P. M. Sobre el concepto de salud enfermedad: descripción y explicación de la situación de salud. Boletín Epidemiológico Organización Panamericana de la Salud, Washington, DC, v. 10, n. 4, p. 1-7, 1990.

GUZMÁN CASTIBLANCO, H. I. Cambios en el paisaje de ciudad bolivar por la explotación de canteras en el periodo 2000-2014. 2014. 37 f. Trabalho de Conclusão de Curso (Especialista en Planeación Ambiental y Manejo Integral de los Recursos Naturales). Facultad de Ingeniería, Universidad Militar Nueva Granada, Bogotá. 2015.

HERRERA DURÁN, N. No le saque la piedra a la montaña. El espectador, [online], 7 fev. 2015. Disponible en: <http://www.elespectador.com/noticias/bogota/no-le-saque-piedra-montana-articulo-542701>. Acceso en: 14 marzo 2017.

INSUASTY RODRÍGUEZ, A.; GRISALES, D.;

GUTIÉRREZ LEÓN, E. M. Conflictos asociados a la gran minería en Antioquia. Ágora U.S.B, Medellin, v. 13, v. 2 , p. $279-539,2013$.
MCS EN CONTACTO. Autostop: diario de un aventurer@. Disponible en: <http://mcsencontactoprensa. blogspot.com.br/p/exploradores.html>. Acceso en: 14 marzo 2017.

\section{PROGRAMA DE LAS NACIONES UNIDAS PARA} EL MEDIO AMBIENTE (PNUMA). Oficina Regional para América Latina y el Caribe. Tendencias del flujo de materiales y productividad de recursos en américa latina. Panamá: Pnuma, 2013. Disponible en: <http://www. pnuma.org/MFA_espanol\%20low\%20res.pdf >. Acceso en: 14 marzo 2016.

\section{SACCHI, M.; HAUSBERGER, M.; PEREYRA, A.}

Percepción del proceso salud-enfermedad-atención y aspectos que influyen en la baja utilización del sistema de salud, en familias pobres de la ciudad de salta. Salud colectiva, Lanús, v. 3, n. 3, p. 271-283, 2007.

SALAMANCA, L. J. G. (Coord.). Minería en Colombia: fundamentos para superar el modelo extractivista. Bogotá: Contraloría General de la Republica, 2013. Disponible en: <http://lasillavacia.com/sites/default/ files/mineropedia/mineria_en_colombia.pdf $>$. Acceso en: 14 marzo 2013.

Recebido para publicación en agosto de 2016

Versión final en marzo de 2017

Conflicto de intereses: inexistente

Apoyo financiero: no hubo 